\title{
Estimación y variabilidad temporal de talla de madurez gonadal de la caballa (Scomber japonicus peruanus) en el litoral peruano
}

\section{Estimation and temporal variability in size at gonad maturity of Chub Mackerel (Scomber japonicus peruanus) in the Peruvian littoral}

\section{Josymar Torrejón-Magallanes*, Javier Sánchez, Julio Mori, Marilú Bouchon y Miguel Ñiquen}

Instituto del Mar del Perú, Esquina Gamarra y General Valle S/N, Chucuito, Callao, Perú.

* Autor para correspondencia

Email Josymar Torrejón-Magallanes: jotorrejon@imarpe.gob.pe

Email Javier Sánchez: jsanchez@imarpe.gob.pe

Email Julio Mori: jmori@imarpe.gob.pe

Email Miguel Ñiquen: mniquen@imarpe.gob.pe

Email Marilú Bouchon: mbouchon@imarpe.gob.pe

\begin{abstract}
Resumen
Se estimaron valores de la talla de madurez gonadal $\left(L_{50}\right)$ de la caballa (Scomber japonicus peruanus) para los años $1994-2017$ en el litoral peruano. El análisis se realizó para el periodo noviembre - marzo donde ocurre la mayor actividad reproductiva. La proporción de hembras maduras en relación a la talla se ajustó mediante un modelo logístico binomial, para ello se utilizaron modelos lineales generalizados con efectos mixtos (MLGM) que asumieron efectos aleatorios asociados a los periodos. El ajuste con el MLGM, mediante sus efectos fjos estimó una $L_{50}$ de $24.8 \mathrm{~cm}$ de longitud a la horquilla (LH) $(24.69 \mathrm{~cm}-24.9 \mathrm{~cm})$, mientras que con cambios entre periodos, mediante sus efectos aleatorios, los valores oscilaron entre $20.4 \mathrm{~cm}$ y $27.0 \mathrm{~cm}$ de LH en promedio. Se observó que un aumento en la temperatura causó la disminución de la $L_{50}$ en los periodos 1997 - 1999 y 2015 - 2016, los cuales coinciden con la presencia de eventos EI Niño. Una correlación positiva fue encontrada entre las $L_{50}$ y la biomasa desovante, estos cambios en las $L_{50}$ podrían indicar un efecto de denso-dependencia. No se mostró una tendencia en la serie de $L_{50}$, por tanto no habría un efecto de la presión de pesca, traducida como la tasa instantánea de mortalidad por pesca $(F)$ sobre la $L_{50}$. Sin embargo, creemos que estas disminuciones de la L50, en relación a una baja densidad poblacional y principalmente a un incremento en la $\mathrm{F}$ deben darse como un efecto a largo plazo y no en eventos puntuales como se reflejaron en nuestros resultados.
\end{abstract}

Palabras clave: Caballa; talla de madurez gonadal; cambios temporales; modelos lineales generalizados mixtos; efectos aleatorios.

\section{Abstract}

The values of size-at-gonad maturity $\left(L_{50}\right)$ of chub mackerel (Scomber japonicus peruanus) were estimated for the years $1994-2017$ in the Peruvian littoral. The analysis was performed for the period November - March where the highest reproductive activity occurs. The proportion of mature females was ftted using a logistic binary model, through generalized linear mixed models (MLGM), which assumes random effects associated with the periods. The GLMM, with fixed effects estimated a $L_{50}$ of $24.8 \mathrm{~cm}$ of fork length (FL) $(24.69 \mathrm{~cm}-24.9 \mathrm{~cm})$, while with changes between periods, with random effects, $L_{50}$ ranged between $20.4 \mathrm{~cm}$ and $27.0 \mathrm{~cm}$ FL. It was observed that an increase in temperature caused the decrease of the $L_{50}$ in the years $1997-1999$ and 2015 - 2016, which coincides with El Niño events. A positive correlation was found between the $L_{50}$ and spawning biomass, so changes in $L_{50}$ may be a densitydependent effect. There was no trend in the $L_{50}$ series, so there would be no effect of the fishing mortality (F) on the $L_{50^{\circ}}$. However, we think that the decrease in the $L_{50}$ in relation to a low population density and mainly an increase in $F$ should be given as a long-term effect and not in isolated events as our results showed.

Keywords: Chub mackerel; size-at-gonad maturity; temporary changes; generalized linear mixed models; random effects.

Citación:

Torrejón-Magallanes J., J. Sánchez, J. Mori, M. Bouchon \& M. Ñiquen. 2017. Estimación y variabilidad temporal de talla de madurez gonadal de la caballa (Scomber japonicus peruanus) en el litoral peruano. Revista peruana de biología 24(4): 391 - 400 (diciembre 2017). doi: http://dx.doi. org/10.15381/rpb.v24i4.13741

Presentado: $\quad 18 / 08 / 2017$

Aceptado: $\quad 19 / 11 / 2017$

Publicado online: $14 / 12 / 2017$
Información sobre los autores:

JT-M, JS JM: realizaron el diseño experimental; analizaron los datos; JT-M: redactó el manuscrito; JT-M, JS, JM, MB, MÑ: revisaron y aprobaron el manuscrito

Los autores no incurren en conflictos de intereses. 


\section{Introducción}

Asignar un valor para representar la edad o talla de madurez y probar diferentes metodologías para estimarlo ha sido una tarea de interés por mucho tiempo para investigadores pesqueros. La talla de madurez, definido como la talla a la cual el $50 \%$ de los individuos son maduros (Roa et al. 1999), es una de las informaciones más importantes que se necesitan conocer de una población. Esta talla se obtiene habitualmente a partir de las frecuencias de peces maduros a lo largo de un segmento específico de una distribución de edad o de talla (Trippel \& Harvey 1991). Esta información, constituye uno de los parámetros reproductivos básicos en la evaluación y manejo de recursos pesqueros explotados comercialmente, ya que determina la ojiva de madurez que permite separar la fracción madura de un stock de peces (Cubillos \& Alarcón 2010). Además, la talla de madurez es usada como punto de referencia para establecer la talla mínima de captura o desembarque (Corgos \& Freire 2006), la cual permite evitar la sobrepesca sobre individuos juveniles que aún no se han reproducido al menos una vez. Otro punto importante es que la talla de madurez se ha propuesto como indicador del nivel poblacional por efecto de las pesquerías (Rochet \& Trenkel 2003), ya que se ha demostrado que la presión de pesca intensa y sostenida induce la maduración temprana de los recursos explotados (Trippel 1995).

Se han reportado diferentes métodos para estimar la talla de madurez, así se menciona: a través del índice gonadosomático (IGS), relación longitud-peso, mediante criterios macro y/o microscópico de las condiciones de las gónadas clasificando los peces maduros en función de las características histológicas funcionales o estructurales y a través de parámetros del consumo relativo de oxígeno (p.e. Oliva et al. 1986, Fontoura et al. 2009, Cubillos \& Alarcón 2010, Fontoura et al 2010). En el Perú, los trabajos referidos a la estimación de la talla de madurez de las especies comercialmente explotadas, están basados principalmente en la catalogación macro o microscópica del estadio de madurez gonadal a través de un rango de tallas ajustándolas a una función logística, así existen estudios en anchoveta Engraulis ringens (Pauly \& Soriano 1987), jurel Trachurus murphyi (Dioses et al. 1988, Perea et al. 2013) y caballa Scomber japonicus peruanus (Mendo 1984).

La caballa Scomber japonicus peruanus es una especie con amplia distribución en el Pacífico Sur, encontrándose en las costas de Ecuador, Perú, Chile y Costa Rica (Chirichigno \& Velez 1998). En el litoral peruano esta especie forma grandes cardúmenes (Caramantin-Soriano 2001) y se encuentra asociada a Aguas Ecuatoriales Superficiales (Dioses et al. 2002). Así mismo, soporta una importante pesquería en el litoral peruano la cual es explotada principalmente por la flota industrial de cerco y es usada para el consumo humano directo. Sus desembarques máximos se dieron a fines de los 90's con 380 mil toneladas, siendo las magnitudes promedio en los últimos 3 años alrededor de las 90 mil toneladas (PRODUCE 2017). Los estudios de esta especie en el Perú estuvieron relacionados principalmente a su crecimiento, mortalidad (Mendo 1984, Caramantín-Soriano et al. 2008), fecundidad (Peña et al. 1986, Buitrón y Perea, 1998) y algunos aspectos reproductivos (Caramantin-Soriano 2001, Caramantin-Soriano et al. 2009). En cuanto a estudios relacionados a la estimación de talla de madurez y de desove se tiene el trabajo de Mińano y Castillo (1972) y Mendo (1984).
El objetivo del presente trabajo es proveer información de la talla de madurez gonadal $\left(L_{50}\right)$ a partir de las catalogaciones macroscópicas de las gónadas de caballa (Scomber japonicus peruanus); además, analizar los cambios temporales en la $L_{50}$ mediante el uso de Modelos Lineales Generalizados Mixtos (MLGM). Así mismo, discutir acerca de los cambios de la $L_{50}$ en su relación con la temperatura superficial del mar y posibles efectos de denso-dependencia y presión de pesca a través de los niveles de biomasa desovante y la tasa instantánea de mortalidad por pesca $(\mathrm{F})$ respectivamente. Esta información contribuirá a un mayor conocimiento sobre la biología reproductiva de esta especie, así mismo ayudará a establecer y/o mejorar medidas de manejo y ordenación para dicho recurso.

\section{Materiales y métodos}

La información para este estudio provino del programa de seguimiento de la pesquería que ejecuta el Instituto del Mar del Perú (IMARPE), para ello se empleó el protocolo de muestreo propuesto por Bouchon et al. (2001). El periodo analizado constó de 24 años (1994 - 2017) y abarcó las zonas del litoral peruano entre los $3^{\circ} 23^{\prime} \mathrm{S}$ y $18^{\circ} 21^{\prime} \mathrm{S}$.

Los datos para la estimación de la $L_{50}$ consistieron en la longitud a la horquilla $(\mathrm{LH})$ en centímetros y los estadios de madurez gonadal de las hembras de caballa. Estos estadios se catalogaron según el criterio de Johansen (1924) y se clasificaron en dos grupos: inmaduras y maduras. Así, según lo establecido por Buitrón et al. (2011), se consideró como hembras inmaduras a aquellas con estadios I - II y maduras III - VIII. Así mismo, para el análisis, solamente se incluyeron los meses entre noviembre y marzo, momento en el cual ocurre la mayor actividad reproductiva (Caramantin-Soriano et al. 2009). Este periodo se consideró como la principal época reproductiva de la caballa.

Las proporciones de hembras maduras por intervalo de longitud fueron ajustadas mediante un modelo lineal generalizado (GLM) con familia binomial y enlace logit, mediante la siguiente ecuación:

$$
\operatorname{logit}=\log \left(\frac{p}{1-p}\right)(E c .1)
$$

donde: $p$ es la probabilidad de "éxito" y 1- $p$ la probabilidad de "fracaso". En este análisis, "éxito" se define como "maduro" y "fracaso" como "inmaduro". Así construimos un modelo lineal:

$$
\begin{gathered}
\operatorname{logit}(p)=\beta_{0}+\beta_{1} * X(E c .2) \\
\log \left(\frac{p}{1-p}\right)=\beta_{0}+\beta_{1} * X(E c .3)
\end{gathered}
$$

Donde $X$ es la variable independiente $(\mathrm{LH}), \hat{\beta}_{0}$ y $\hat{\beta}_{1}$ son estimaciones del intercepto y la pendiente respectivamente.

El valor de $L_{50}$ es estimado de la siguiente manera:

$$
L_{50}=-\frac{\hat{\beta}_{0}}{\hat{\beta}_{1}}(E c .4)
$$

Para la estimación de la $\hat{\beta}_{0}$ y $\hat{\beta}_{1}$ sin efectos entre periodos, se ajustó mediante la ecuación 2 , agregando los datos de todos 
los años de estudio, para ello se utilizó el paquete "sizeMat" (Torrejón-Magallanes 2016) del software R.

Para los cambios de la $L_{50}$ a través de la serie de tiempo, se asumió que ocurren efectos aleatorios entre periodos. Dichos cambios son la expresión proveniente de un modelo poblacional en que la talla de madurez es un proceso poblacional con parámetros fijos, y que las variaciones entre periodos se deben a efectos aleatorios que podrían ser debido a varios factores asociados. Para ello, se realizó un modelo lineal generalizado de efectos mixtos mediante el paquete "Ime4" de Bates et al. (2015) del software R, en donde las proporciones de individuos maduros se modelaron con pendiente fija e intercepto aleatorio (Modelo 1) y con pendiente e intercepto aleatorios (Modelo 2) de la siguiente manera:

$$
\text { Modelo 1: } P_{i, j} \sim \beta_{0}+\beta_{0_{j}}+\beta_{1} * L_{i, j}+\varepsilon_{i, j}(E c .5)
$$$$
\text { Modelo 2: } P_{i, j} \sim \beta_{0}+\beta_{0 j}+\left(\beta_{1}+\beta_{1 j}\right) * L_{i, j}+\varepsilon_{i, j}
$$

Donde $P_{i, j}$ es la proporción de individuos maduros de la longitud $i$ respecto del total en el periodo $j ; \beta_{0}$ y $\beta_{1}$ son el intercepto y la pendiente general respectivamente, $\beta_{0 j} \sim N\left(0, \sigma^{2}\right)$ es el intercepto aleatorio para cada uno de los periodos $j$, $\beta_{1 j} \sim N\left(0, \sigma^{2}\right)$ es la pendiente aleatoria para cada uno de los periodos $j$ cuyo efecto modifica la pendiente general en función de la variable respuesta, $\mathrm{L}_{\mathrm{i}, \mathrm{j}}$ es la longitud del individuo $i$ en el periodo $j$, y $\varepsilon_{i, j} \sim N\left(0, \sigma^{2}\right)$ es el error aleatorio. El mejor modelo, fue seleccionado con el Criterio de Información de Akaike (AIC).

Para probar efectos de denso-dependencia y presión de pesca sobre las $L_{50}$, se utilizaron los valores de biomasa desovante (BD) y la tasa instantánea de mortalidad por pesca $(F)$ respectivamente, los cuales se obtuvieron del modelo Joint Jack Mackerel (JJM) aplicado al recurso caballa. Así mismo, se relacionó la serie de las $L_{50}$ con la Temperatura Superficial del Mar (TSM) promedio frente a las costas peruanas entre las 10 y 80 millas de distancia a la costa, las cuales se obtuvieron del sistema ERDAP (Simons 2011).

\section{Resultados}

Durante el periodo 1994 - 2017 se contaron 25912 hembras de las cuales 6063 fueron inmaduras y 19849 maduras. La mediana de las longitudes de las hembras inmaduras osciló entre 23 y $28 \mathrm{~cm}$ de $\mathrm{LH}$ mientras que para las hembras maduras osciló entre 27 y $32 \mathrm{~cm}$ de LH (Fig. 1, Tabla 1). Así mismo, se observó que durante los periodos 1997 - 2000 y 2014 - 2016, la mediana de los individuos maduros fue menor con respecto a los demás periodos con valores de 27 y $28 \mathrm{~cm}$ de LH.

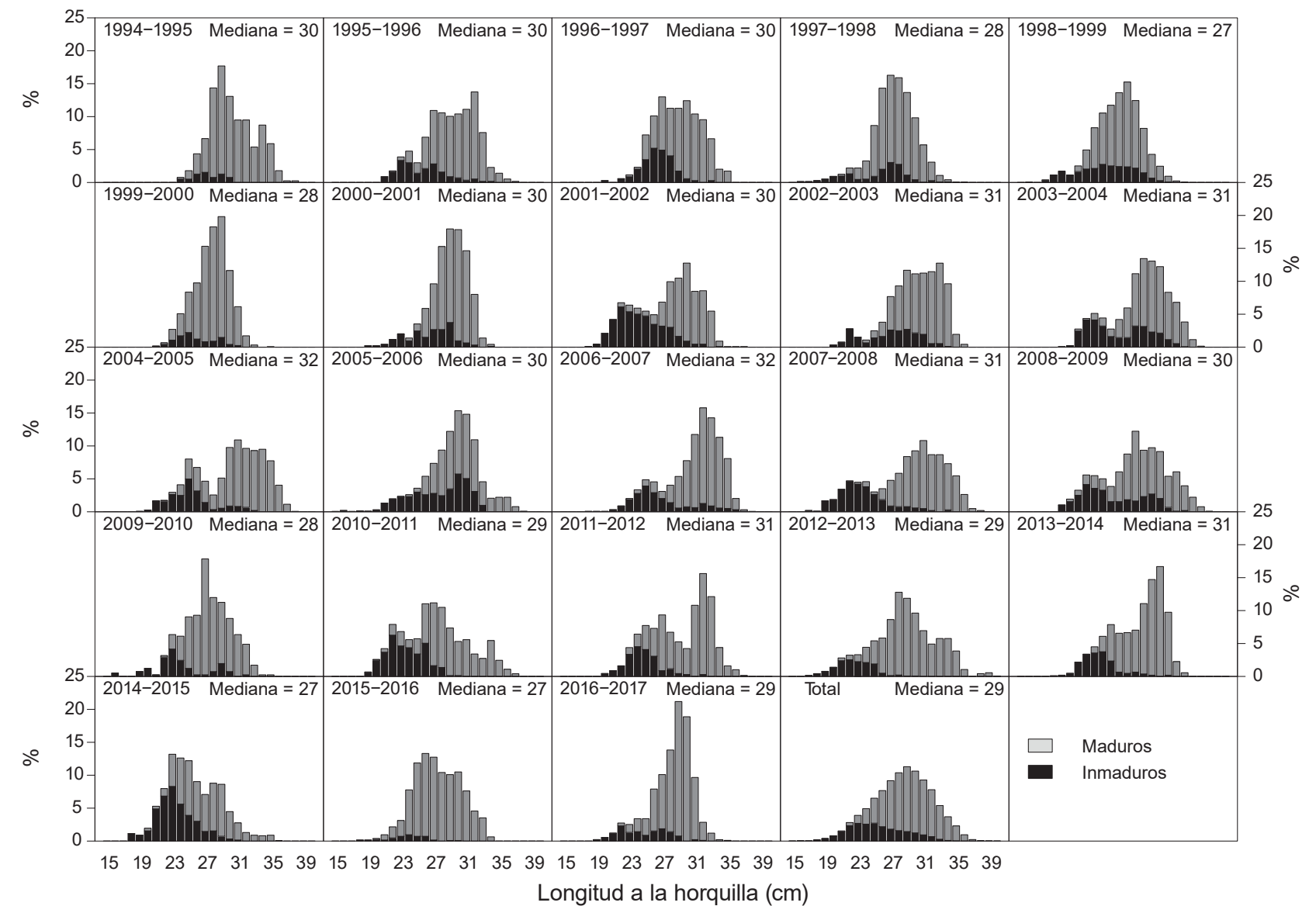

Figura 1. Distribución de longitudes (porcentajes) de caballas (Scomber japonicus peruanus) hembras inmaduras (gris oscuro) y maduras (gris claro) durante 1995 - 2017. Se muestra el valor de la mediana de la longitud a la horquilla de las hembras maduras. 
Tabla 1. Tamaño de muestra, mediana, mínimo y máximo de la longitud a la horquilla (LH) de hembras de caballa (Scomber japonicus peruanus) inmadura y madura por periodo (noviembre - marzo).

\begin{tabular}{|c|c|c|c|c|c|}
\hline \multirow{2}{*}{$\begin{array}{c}\text { Periodo } \\
\text { (Nov - Mar) }\end{array}$} & \multicolumn{2}{|c|}{ Inmaduras } & \multicolumn{2}{|c|}{ Maduras } & \multirow[b]{2}{*}{ Total } \\
\hline & $\mathbf{N}$ & $\begin{array}{c}\text { LH (cm) } \\
\text { mediana (min-max) }\end{array}$ & $\mathbf{N}$ & $\begin{array}{c}\text { LH (cm) } \\
\text { mediana (min-max) }\end{array}$ & \\
\hline 1994 - 1995 & 26 & $27(24-30)$ & 364 & $30(24-38)$ & 390 \\
\hline 1995 - 1996 & 111 & $25(21-34)$ & 456 & $30(22-37)$ & 567 \\
\hline 1996 - 1997 & 84 & $26(20-33)$ & 262 & $30(23-35)$ & 346 \\
\hline 1997 - 1998 & 341 & $26(16-34)$ & 1796 & $28(18-36)$ & 2137 \\
\hline 1998 - 1999 & 523 & $25(16-33)$ & 1582 & $27(19-35)$ & 2105 \\
\hline $1999-2000$ & 130 & $25(21-31)$ & 1091 & $28(22-35)$ & 1221 \\
\hline $2000-2001$ & 187 & $27(19-34)$ & 749 & $30(23-34)$ & 936 \\
\hline $2001-2002$ & 452 & $24(18-34)$ & 645 & $30(19-37)$ & 1097 \\
\hline $2002-2003$ & 204 & $27(20-34)$ & 721 & $31(22-36)$ & 925 \\
\hline $2003-2004$ & 409 & $26(20-35)$ & 900 & $31(22-37)$ & 1309 \\
\hline $2004-2005$ & 321 & $25(19-33)$ & 1164 & $32(21-38)$ & 1485 \\
\hline $2005-2006$ & 771 & $28(15-33)$ & 1268 & $30(23-38)$ & 2039 \\
\hline $2006-2007$ & 345 & $26(18-37)$ & 1239 & $32(23-38)$ & 1584 \\
\hline $2007-2008$ & 291 & $23(17-34)$ & 735 & $31(23-38)$ & 1026 \\
\hline $2008-2009$ & 317 & $25(20-35)$ & 721 & $30(20-38)$ & 1038 \\
\hline 2009 - 2010 & 72 & $23(16-32)$ & 337 & $28(22-35)$ & 409 \\
\hline $2010-2011$ & 247 & $23(19-30)$ & 487 & $29(19-37)$ & 734 \\
\hline $2011-2012$ & 144 & $24(20-32)$ & 541 & $31(23-37)$ & 685 \\
\hline $2012-2013$ & 211 & $24(17-30)$ & 1213 & $29(20-40)$ & 1424 \\
\hline $2013-2014$ & 197 & $24(19-33)$ & 870 & $31(24-35)$ & 1067 \\
\hline $2014-2015$ & 496 & $23(18-31)$ & 733 & $27(20-36)$ & 1229 \\
\hline 2015 - 2016 & 58 & $24(18-30)$ & 1190 & $27(20-34)$ & 1248 \\
\hline $2016-2017$ & 126 & $24(19-32)$ & 785 & $29(22-35)$ & 911 \\
\hline Total & 6063 & $25(15-37)$ & 19849 & $29(18-40)$ & 25912 \\
\hline
\end{tabular}

El ajuste del MLG para la totalidad de los datos estimó una $L_{50}$ de $24.4 \mathrm{~cm}(22.9 \mathrm{~cm}-25.9 \mathrm{~cm})$ de LH (Fig. 2). Los parámetros del modelo se muestran en la Tabla 2.

Para los cambios entre periodos con los MLGM, el Modelo 2 , con intercepto y pendiente aleatorio, presentó un mejor desempeño según el AIC (= 19043). Los parámetros del modelo se muestran en la Tabla 3. Este modelo mediante sus efectos fijos

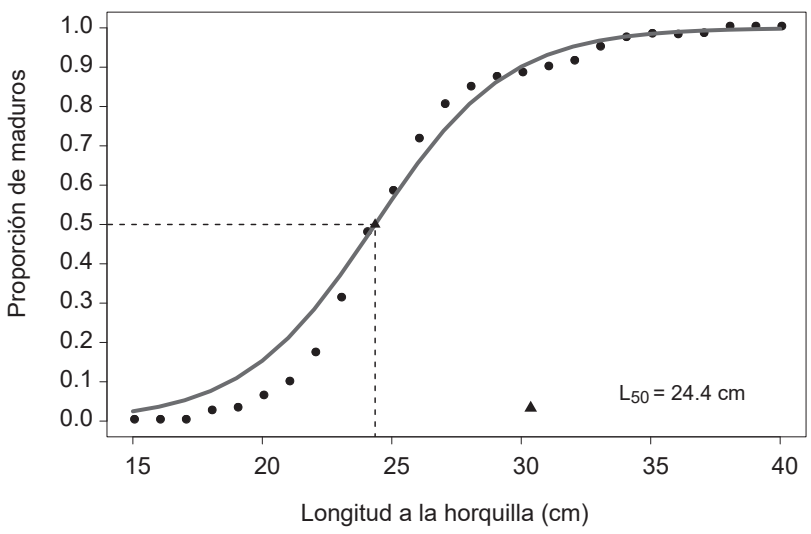

Figura 2. Proporción de hembras maduras de caballa (Scomber japonicus peruanus) en función de la longitud a la horquilla para el periodo $1994-2017$. estimó un $L_{50}$ de $24.8 \mathrm{~cm}$ de LH $(24.69-24.90 \mathrm{~cm})$, mientras que con cambios entre periodos, mediante los efectos aleatorios, los valores oscilaron entre 20.4 y $27.0 \mathrm{~cm}$ de LH (Tabla 3 y Fig. 3). La varianza del efecto aleatorio asociada al intercepto y a la pendiente fue de $\sigma_{\beta_{0_{j}}}^{2}=16.84$ y $\sigma_{\beta_{1_{j}}}^{2}=0.026$.

\section{Discusión}

Para el litoral peruano, el primer trabajo relacionado a la estimación de la talla de madurez de caballa fue realizado por Mińano y Castillo (1971), quienes para la zona de Chimbote reportaron una talla de madurez de $27 \mathrm{~cm}$ y $28 \mathrm{~cm}$ de longitud total (LT) para las hembras y los machos respectivamente, encontrando además que la talla más frecuente a la que se realiza el desove fue de $31.5 \mathrm{~cm}$ de LT. Chávez (1973) estimó para el área de Paita una talla de madurez de $37.5 \mathrm{~cm}$ de longitud total.

Tabla 2. Coeficientes y $L_{50}$ estimado del modelo lineal generalizado para el periodo $1994-2017$

\begin{tabular}{cccccc}
\hline Coeficientes & Estimación & $\begin{array}{c}\text { Error } \\
\text { estandar }\end{array}$ & Valor Z & $\operatorname{Pr}(>|\mathbf{z}|)$ & \\
\hline Intercepto & -9.549283 & 0.157608 & -60.59 & $<0.05$ & $* * *$ \\
Pendiente & 0.392063 & 0.005877 & 66.71 & $<0.05$ & $* * *$ \\
$L_{50}(\mathbf{c m})$ & 24.4 & - & - & - & \\
\hline
\end{tabular}




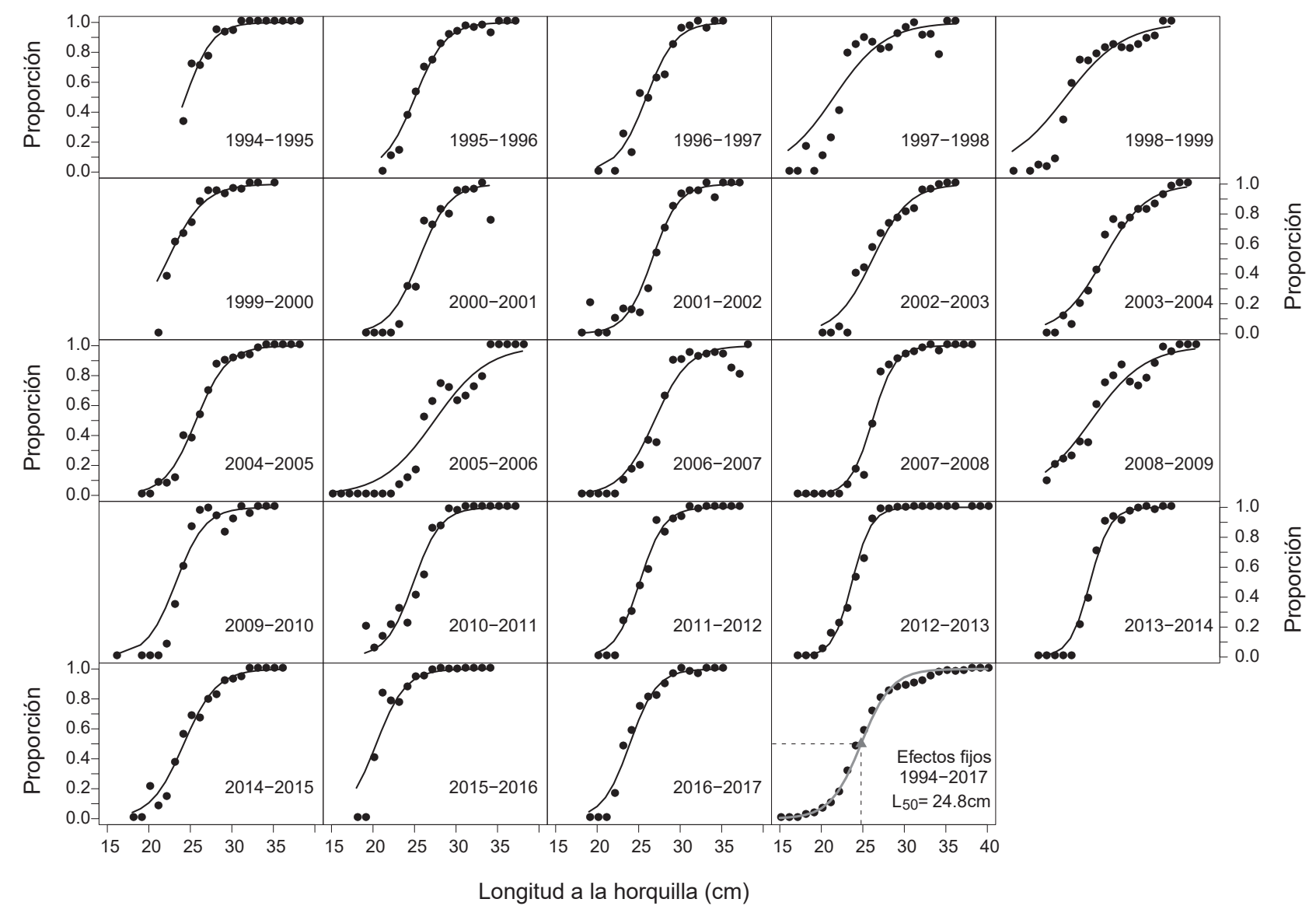

Figura 3. Estimación temporal de la $L_{50}$ de las hembras de caballa (Scomber japonicus peruanus) del Modelo 2 con intercepto y pendiente aleatorio para el periodo $1994-2017$.

Tabla 3. Coeficientes y $L_{50}$ estimados del Modelo 2 con intercepto y pendiente aleatorio para el periodo $1994-2017$.

\begin{tabular}{|c|c|c|c|c|c|}
\hline Coeficientes & Estimación & Error estandar & Valor Z & $\operatorname{Pr}(>|z|)$ & $\mathrm{L}_{50}(\mathrm{LH} \mathrm{cm})$ \\
\hline \multicolumn{6}{|l|}{ Efectos fijos } \\
\hline Intercepto & -13.47726 & 0.8718 & -15.46 & $<0.05$ & \multirow{2}{*}{24.8} \\
\hline Pendiente & 0.54362 & 0.03393 & 16.02 & $<0.05$ & \\
\hline Efectos aleatorios & \multicolumn{2}{|c|}{ Intercepto aleatorio } & \multicolumn{2}{|c|}{ Pendiente aleatorio } & $\mathrm{L}_{50}(\mathrm{LH} \mathrm{cm})$ \\
\hline $1994-1995$ & \multicolumn{2}{|c|}{-14.933} & \multicolumn{2}{|c|}{0.611} & 24.4 \\
\hline 1995 - 1996 & \multicolumn{2}{|c|}{-13.602} & \multicolumn{2}{|c|}{0.543} & 25.1 \\
\hline $1996-1997$ & \multicolumn{2}{|c|}{-14.729} & \multicolumn{2}{|c|}{0.568} & 25.9 \\
\hline $1997-1998$ & \multicolumn{2}{|c|}{-6.925} & \multicolumn{2}{|c|}{0.322} & 21.5 \\
\hline $1998-1999$ & \multicolumn{2}{|c|}{-6.379} & \multicolumn{2}{|c|}{0.285} & 22.4 \\
\hline $1999-2000$ & \multicolumn{2}{|c|}{-10.294} & \multicolumn{2}{|c|}{0.461} & 22.3 \\
\hline $2000-2001$ & \multicolumn{2}{|c|}{-14.082} & \multicolumn{2}{|c|}{0.551} & 25.6 \\
\hline $2001-2002$ & \multicolumn{2}{|c|}{-16.728} & \multicolumn{2}{|c|}{0.628} & 26.6 \\
\hline $2002-2003$ & \multicolumn{2}{|c|}{-11.908} & \multicolumn{2}{|c|}{0.455} & 26.2 \\
\hline $2003-2004$ & \multicolumn{2}{|c|}{-10.495} & \multicolumn{2}{|c|}{0.389} & 27.0 \\
\hline $2004-2005$ & \multicolumn{2}{|c|}{-13.714} & \multicolumn{2}{|c|}{0.534} & 25.7 \\
\hline $2005-2006$ & \multicolumn{2}{|c|}{-8.507} & \multicolumn{2}{|c|}{0.310} & 24.4 \\
\hline $2006-2007$ & \multicolumn{2}{|c|}{-13.103} & \multicolumn{2}{|c|}{0.487} & 26.9 \\
\hline $2007-2008$ & \multicolumn{2}{|c|}{-19.289} & \multicolumn{2}{|c|}{0.737} & 26.2 \\
\hline $2008-2009$ & & & & & 25.5 \\
\hline $2009-2010$ & & 247 & & & 23.3 \\
\hline $2010-2011$ & & 283 & & & 24.9 \\
\hline $2011-2012$ & & 217 & & & 25.1 \\
\hline 2012 - 2013 & & 379 & & & 23.7 \\
\hline $2013-2014$ & & 892 & & & 25.4 \\
\hline $2014-2015$ & & 268 & & & 24.2 \\
\hline 2015 - 2016 & & 542 & & & 20.4 \\
\hline $2016-2017$ & & 717 & & & 23.9 \\
\hline
\end{tabular}


Por último, Mendo (1984) reportó tallas medias de madurez que oscilaron entre $32.25-33.55 \mathrm{~cm}$ y $33.4-34.2 \mathrm{~cm}$ para las áreas de Callao y Chimbote respectivamente, así mismo, menciona que para ambas zonas, esta especie inicia su madurez entre los $26-28 \mathrm{~cm}$ de LT. En el presente trabajo la talla de madurez gonadal utilizando el conjunto de datos para el periodo 1994 - 2017 fue de $24.4 \mathrm{~cm}(22.9 \mathrm{~cm}-25.9 \mathrm{~cm})$ de longitud a la horquilla (LH). Las diferencias con los valores estimados previamente, se podrían deber a que para este estudio, el periodo mensual considerado para el análisis fue de noviembre - marzo, el cual se consideró el principal periodo de actividad reproductiva, al agrupamiento de los estadios de madurez gonadal para catalogar a los individuos inmaduros y maduros, al rango de tallas utilizadas en la estimación y a la metodología estadística de estimación. Así también, las estimaciones de estudios previos se reportan en LT mientras que este trabajo está basado en la LH.

Para mostrar los cambios entre periodos en las $L_{50}$ se utilizó un modelo lineal generalizado de efectos mixtos (MLGM), el cual, a diferencia de usar un Modelo Lineal Generalizado (MLG) por año para la estimación, asume que esta talla es un proceso poblacional con parámetros fijos, y que las variaciones entre los periodos se deben básicamente a efectos aleatorios. Estos efectos aleatorios pueden estar relacionados al muestreo (época y lugar), a variables físicas, factores de denso-dependencia/independencia (cambios en la magnitud de biomasa desovante, reclutamiento), cambios en el crecimiento o al truncamiento de la estructura de tallas por la presión de pesca ejercida (Bustos \& Cubillos 2016).
La serie de tiempo de las $L_{50}$ mostró cambios aleatorios con los valores más bajos en el periodo entre 1997 - 2000 y 2015 2016 (Fig. 4), periodos en los cuales las medianas de las tallas de los individuos maduros fueron las menores con valores entre 27 y $28 \mathrm{~cm}$ de LH lo que pudo determinar que las tallas de madurez sean las menores en la serie de tiempo (Tabla 1). Sin embargo, para el periodo 1999 - 2000 y 2015 - 2016, esto puede atribuirse también a la poca representatividad de individuos inmaduros en la muestra (Figs. 1 y 3).

Se ha descrito que la temperatura juega un rol fundamental en la biología reproductiva de los peces ya que esto afecta la tasa metabólica y su ciclo reproductivo (Jian et al. 2003, Palomares-García 2003, Niquen \& Bouchon 2004). Para este estudio, se observó que un aumento en la TSM causó una disminución de la $L_{50}$ en los periodos 1997 - 1998, 1998 1999 y 2015 - 2016, los cuales coinciden con la presencia de eventos El Niño (Fig. 5a). Este efecto ha sido documentado en varios trabajos, donde señalan que la talla y edad de madurez se ve afectada por la temperatura (Atkinson 1994, Berringan \& Charnov 1994), así a temperaturas altas la madurez se alcanza a tallas menores (Fisher 1999, Jonsson et al. 2012, Yoneda et al. 2015). Además, podría haber un efecto del crecimiento en la madurez (Berringan \& Charnov 1994, Grover 2005, Jonsson et al. 2012), así Heino et al. (2002) señala que un incremento en la tasa de crecimiento de juveniles junto con la temperatura puede resultar en una disminución en la longitud y/o edad de madurez. En el mar peruano, Niquen y Bouchon (2004) reportaron que la actividad reproductiva de $S$. japonicus peruanus fue

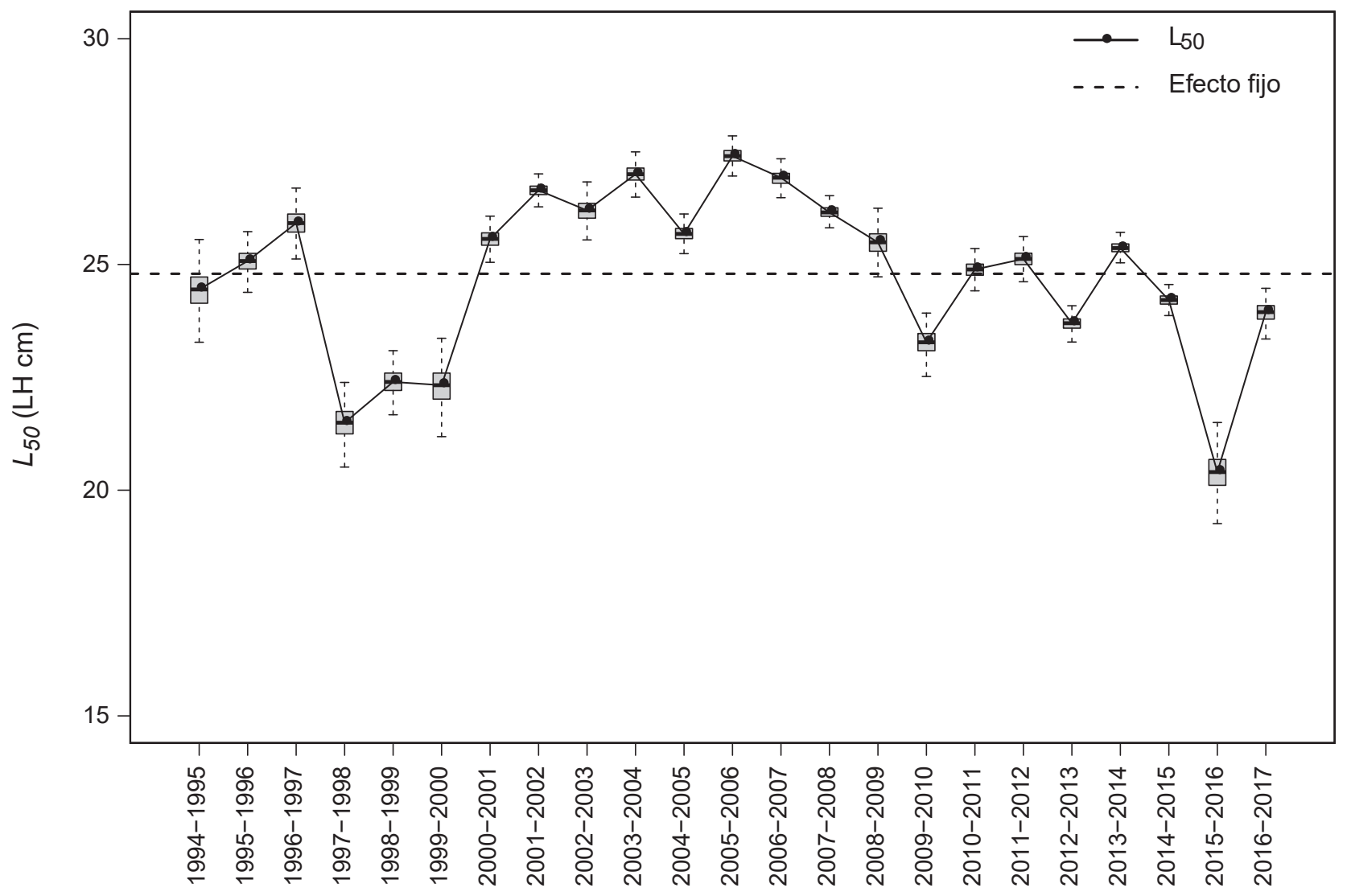

Figura 4. Serie de tiepo de la estimación temporal de la $L_{50}$ de las hembras de caballa (Scomber japonicus peruanus) del Modelo 2 con intercepto y pendiente aleatorio para el periodo $1994-2017$. La línea horizontal discontinua muestra el valor de la $L_{50}$ estimada mediante los efectos fijos del modelo. 
más intensa durante el inicio del evento El Niño 1997 - 1998 y que individuos juveniles se encontraban desovando al final de este evento. Así mismo, Caramantin et al. (2009) sugiere que la presencia de El Niño 1992-1993 tuvo influencia en la actividad reproductiva de la caballa. Aunque estos estudios no mencionan directamente una disminución en la talla de madurez en función de eventos anómalos, es posible que estos cambios se hayan producido en dichos periodos ya que se ha reportado este comportamiento en otras especies pelágicas en el mar peruano. Así, Perea (1998) muestra que durante El Niño 1997 - 1998 la sardina (Sardinops sagax sagax) presentó una maduración a una edad más temprana probablemente por las condiciones creadas por El Niño 1997 - 1998. Sin embargo, parece que los cambios en la temperatura del agua por sí solo no pueden explicar directamente los cambios observados en la biología reproductiva de algunos peces. Wootton (1984) menciona que un organismo al encontrarse en un inevitable stress tiene como resultado un crecimiento más lento que alteraría su edad y talla de madurez, madurando a una talla más pequeña; además señala que la madurez está ligada tanto genética como ambientalmente teniendo cada uno de ellos una influencia particular. Por tanto, para la caballa, parece ser que el ambiente en periodos El Niño habría sido la que tuvo mayor influencia en el inicio de maduración a tallas menores.

Para comprobar si existen efectos de denso-dependencia se relacionó a las $L_{50}$ con las magnitudes de la biomasa desovante
(BD). Se observó que los valores de $L_{50}$ y $\mathrm{BD}$ estuvieron positivamente correlacionadas (Fig. 5b), con lo que se corroboraría un efecto de denso - dependencia. Esto coincide con lo encontrado por diferentes estudios (Jorgensen 1990, Cardinale \& Modin 1999, Hutchings 2005, Domínguez - Petit 2007), quienes encontraron que los cambios en la talla de madurez estuvieron influenciados por las variaciones en la densidad poblacional (biomasa desovante), en donde una disminución de la abundancia causaba una disminución de la talla de madurez. La explicación de este fenómeno podría deberse a que una reducción en la biomasa podría favorecer un crecimiento acelerado como una respuesta compensatoria (Cardinale \& Modin 1999), lo que conllevaría a una maduración gonadal más temprana.

La serie de las $L_{50}$ no mostraron ninguna tendencia en el periodo analizado. Es conocido que una pesca intensa y sostenida induce la maduración temprana de los recursos explotados. Este cambio en la madurez, es una función de los cambios en las frecuencias del genotipo en una población con el tiempo, por tanto la maduración a una edad más temprana y un tamaño más pequeño puede permitir que los individuos contribuyan en una o dos temporadas de desove antes de ser capturadas (Trippel 1995). A pesar de no haberse observado una tendencia, se relacionó a las $L_{50}$ con la tasa instantánea de mortalidad por pesca $(F)$ (Fig. 5c), encontrándose que en el periodo 1997 2000 se dieron los valores de $F$ más altos y los $L_{50}$ más bajos. Sin embargo, creemos que esta disminución de la $L_{50}$ en dicho
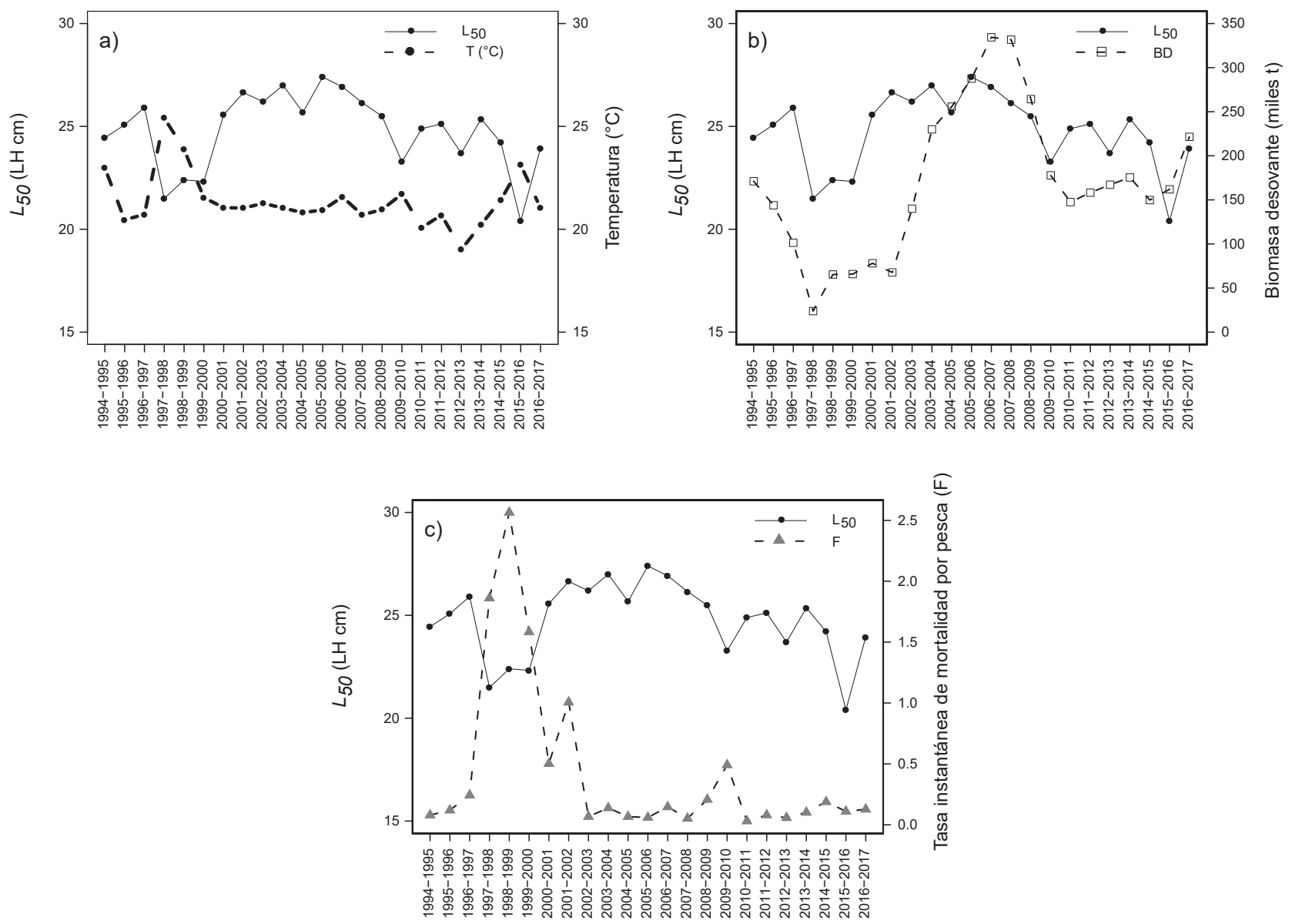

Figura 5. Correlaciones entre las $L_{50}$ vs Temperatura Superficial del Mar(a), Biomasa desovante (b) y la tasa instantánea de mortalidad por pesca (c) para el periodo $1994-2017$ 
periodo, en relación a un incremento en la $F$, debe haberse dado como un efecto a largo plazo y no necesariamente en eventos puntuales como se reflejaron en nuestros resultados. Por otro lado, estos cambios en dichos periodos, también podrían ser producto de una interacción de varios efectos: aumento de temperatura, una baja en la magnitud de BD y altos niveles de $F$, así Trippel (1995) señala que, es más probable que los cambios en la talla de madurez sean una consecuencia de una combinación de factores que actúen conjuntamente y tengan orígenes compensatorios o genéticos.

Sin embargo, se sugiere para futuros trabajos incluir variables físicas (temperatura, salinidad, concentración de clorofila, etc.) y espaciales para modelar con mayor precisión los cambios de la $L_{50^{\circ}}$

\section{Agradecimientos}

El autor desea expresar su agradecimiento a la Dirección General de Investigaciones en Recursos Pelágicos del IMARPE por brindar la información de los muestreos biológicos de caballa. A todas las personas involucradas en el desarrollo del trabajo por sus valiosos comentarios y sugerencias. Así mismo, al Mag. Wencheng Lau - Medrano por proporcionar los datos de Temperatura Superficial del Mar satelital del sistema ERDAP.

\section{Literatura citada}

Atkinson D. 1995. Effects of temperature on the size of aquatic ectotherms: Exceptions to the general rule. Journal of Thermal Biology, Effects of Rising Temperature on the Ecology and Physiology of Aquatic Organisms, 20 (1):61-74. https:// doi.org/10.1016/0306-4565(94)00028-H

Bates D., M. Maechler, B. Bolker \& S. Walker. 2015. Fitting Linear Mixed-Effects Models Using lme4. Journal of Statistical Software, 67(1), 1-48. https://doi.org/10.18637/jss.v067.i01

Berringan D. \& E.L. Charnov 1994. Reaction norms for age and size at maturity in response to temperature: a puzzle for life historians. Oikos, 474-478. https://doi.org/10.2307/3545787

Bouchon M., M. Niquen, J. Mori, A. Echevarría \& S. Cahuín. 2001. Manual de muestreo de la pesquería pelágica. Informe Progresivo. Instituto del Mar del Perú - Callao. 157: 38 pp.

Buitrón B., A. Perea, J. Mori, J. Sánchez \& C. Roque. 2011. Protocolo para estudios sobre procesos reproductivos de peces pelágicos y demersales. Informe, Instituto del Mar del Perú 38(4): 373 - 384. http://biblioimarpe.imarpe.gob.pe:8080/ handle/123456789/2172

Bustos B. \& L.A. Cubillos. 2016. Cambios interanuales en la talla de madurez de sardina común, Strangomera bentincki, en la zona centro-sur de Chile (2007-2012). Revista de biología marina y oceanografía, 51(2), 317-325. https:// doi.org/10.4067/S0718-19572016000200009

Caramantín H. 2001. Efecto de la variabilidad ambiental de los aspectos reproductivos de la caballa Scomber japonicus peruanus (Jordan y Hubb, 1925) en el litoral peruano durante 1990 1998. Tesis de maestría. Facultad de Ingeniería. Universidad Nacional Federico Villareal, Lima, Perú. 136p.

Caramantín-Soriano H., L.A. Vega-Pérez \& M. Niquen. 2008. Growth parameters and mortality rate of the Scomber japonicus peruanus (Jordan \& Hubb, 1925) along the Peruvian Coats, South Pacific. Brazilian Journal of Oceanography, 56(3), 201-210. https://doi.org/10.1590/S167987592008000300005

Caramantin-Soriano H., L.A. Vega-Pérez \& M. Niquen. 2009. The influence of the 1992-1993 el ni-o on the reproductive biology of Scomber japonicus peruanus (Jordán \& Hubb, 1925). Brazilian Journal of Oceanography, 57(4), 263-272. https:// doi.org/10.1590/S1679-87592009000400002

Cardinale M. \& J. Modin. 1999. Changes in size-at-maturity of Baltic cod (Gadus morhua) during a period of large variations in stock size and environmental conditions. Fisheries Research, 41: 285 - 295. https://doi.org/10.1016/S01657836(99)00021-1
Chavez D. 1976. Algunos aspectos biológicos de la "caballa" (Scomber japonicas peruanus J. y H.) en el área de Paita y su relación con el factor de condición. Tesis (Br.) Universidad Nacional Mayor de San Marcos. Programa Académico de Ciencias Biológicas. Lima, Perú.

Chirichigno N.F. \& J. Vélez D. 1998. Clave para identificar los peces marinos del Peru (Seguenda edición, revidada y actualizada). Instituto del Mar del Peru, Publicación especial. 496 p.

Corgos A. \& J. Freire. 2006. Morphometric and gonad maturity in the spider crab Maja brachydactyla: a comparison of methods for estimating size at maturity in species with determinate growth. ICES Journal of Marine Science: Journal du Conseil, 63(5), 851-859. https://doi.org/10.1016/j. icesjms.2006.03.003

Cubillos L. \& C. Alarcón. 2010. Estimación de la talla media de madurez sexual en Trachurus murphyi mediante parámetros del consumo relativo de oxígeno. Latin american journal of aquatic research, 38(2), 178-187. https://doi.org/10.3856/ vol38-issue2-fulltext-2

Dioses T., V.H. Alarcón, M.E. Nakama \& A. Echevarría. 1988. Desarrollo ovocitario, fecundidad parcial y distribución vertical de los cardúmenes en desove del jurel Trachurus murphyi $(\mathrm{N})$. En: Memorias del Simposio Internacional de los Recursos Vivos y las Pesquerías en el Pacífico Sudeste. pp. 287 - 294.

Dioses T., R. Dávalos \& J. Zuzunaga. 2002. El Ni-o 1982-1983 and 1997-1998: Effects on Peruvian Jack Mackerel and Peruvian Chub Mackerel. Investigaciones marinas supl. Symp Valparaíso ago. 2002, 30 (1): 185-87. https://doi.org/10.4067/ S0717-71782002030100077.

Domínguez-Petit R., M. Korta, F. Sabrido - Rey, H. Murua, M. Sainza \& C. Pi-eiro. 2008. Changes in size at maturity of European hake Atlantic populations in relation with stock structure and environmental regimes. Journal of Marine Systems, 71(3), 260-278. https://doi.org/10.1016/j.jmarsys.2007.04.004

Fisher M. 1999. Effect of temperature and salinity on size at maturity of female blue crabs. Transactions of the American Fisheries Society 128, 499 - 506. https://doi.org/10.1577/15488659(1999) 128<0499:EOTASO > 2.0.CO;2

Fontoura N. F., A. S. Braun \& P. C. C. Milani. 2009. Estimating size at first maturity $\left(\mathrm{L}_{5}\right)$ from Gonadossomatic Index (GSI) data. Neotropical Ichthyology, 7(2), 217-222. https://doi. org/10.1590/S1679-62252009000200013

Fontoura N. F., A. S. Jesus, G. G. Larre \& J. R Porto. 2010. Can weight/length relationship predict size at first maturity? A case study with two species of Characidae. Neotropical Ichthyology, 8(4), 835-840. https://doi.org/10.1590/S167962252010005000013

Grover M.C. 2005. Changes in size and age at maturity in a population of kokanee Oncorhynchus nerka during a period of declining growth conditions. Journal of Fish Biology, 66(1), 122-134. https://doi.org/10.1111/j.0022-1112.2005.00587.x

Heino M., U. Dieckmann \& O. R. Godo. 2002. Measuring probabilistic reaction norms for age and size at maturation. Evolution, 56(4), 669 - 678. https://doi. org/10.1111/j.0014-3820.2002.tb01378.x

Hutchings J. A. 2005. Life history consequences of overexploitation to population recovery in Northwest Atlantic cod (Gadus morhua). Canadian Journal of Fisheries and Aquatic Sciences, 62(4), 824-832. https://doi.org/10.1139/f05-081

Jian C.Y., S.Y. Cheng \& J.C. Chen. 2003. Temperature and salinity tolerates of yellowfin sea bream, Acanthopagruslotus, at different salinity and temperature levels. Aquacult. Res., v. 34 , n. 2. p. $175-185$. https://doi.org/10.1046/j.13652109.2003.00800.x

Johansen A.C. 1924. On the summer and auttum spawning herring of the Noeth Sea. Meddelelser fra Kommissionen for Havundersogelser. Serie Fiskeri, Bind VII, Nr. 5119 pp., 15 figs. Kobenhavn.

Jorgensen T. 1990. Long-term changes in age at sexual maturity of Northeast Arctic cod (Gadus morhua L.). Journal du Conseil International pour l'Exploration de la Mer 46, 235-248. https://doi.org/10.1093/icesjms/46.3.235 
Jonsson B., A.G. Finstand \& N. Jonsson. 2012. Winter temperatura and food quality affect age at maturity: an experiment test with Atlantic salmon (Salmon salar). Canadian Journal of Fisheries and Aquatic Sciences, 69(11), 1817 - 1826. https:// doi.org/10.1139/f2012-108

Mendo J. 1984. Edad, crecimiento y algunos aspectos reproductivos y alimenticios de la caballa, Scomber japonicus peruanus. Boletin Instituto del Mar del Perú, 8(4):101-156.

Miñano J. \& J. Castillo. 1971. Primeros resultados de la investigación biológico-pesquera de la "caballa" Scomber japonicus peruanus J. y H. Serie Informes Especiales Instituto del Mar del Perú -Callao No IM-84, p. 1-16.

Niquen M. \& M. Bouchon. 2004. Impact of El Niño events on pelagic fisheries in Peruvian waters. Deep sea research part II: topical studies in oceanography, 51(6), 563-574. https:// doi.org/10.1016/S0967-0645(04)00052-9

Oliva J., W. Carbajal \& A. Tresierra. 1986. Biología reproductiva e histología de gónadas de peces. Editorial Biocience, 151 pp.

Palomares-García R., A. Martínez-López, R. De Silva-Dávila, R. FúnesRodríguez, M.A. Carballido-Carranza, R. Avenda-o-Ibarra, A. Hinojosa-Medina \& G.A. López-Ibarra. 2003. Biological effects of El Niño 1997-98 on a shallow subtropical ecosystem: Bahia Magdalena, Mexico. Geofísica Internacional 42(3):455-466.

Pauly D. \& M. Soriano. 1987. Monthly spawning stock and egg production of Peruvian anchoveta (Engraulis ringens) 19531982. In D. Pauly and I. Tsukayama (editors). The Peruvian anchoveta and its upwelling ecosystem: three decades of change, p. 167-178. ICLARM Studies and Reviews Vol. 15, Manila.

Perea Á. 1998. Variabilidad reproductiva y fecundidad parcial de la sardina, Sardinops sagax sagax, en oto-o 1998. Crucero BIC José Olaya Balandra 9805-06 de Tacna a Máncora. Informe, Instituto del Mar del Perú 137:66-71.

Perea Á., J. Mori, B. Buitrón \& J. Sánchez. 2013. Aspectos reproductivos del jurel Trachurus murphyi. Revista Peruana de Biología 20(1):29-34. https://doi.org/10.15381/rpb.v20i1.2616

Peña N., J. Alheit \& M.E. Nakama. 1986. Fecundidad parcial de la caballa del Perú (Scomber japonicus peruanus). Boletin Instittuto del Mar del Perú 10(4):93-104.
PRODUCE 2017. Boletín del Sector Pesquero. Desenvolvimiento Productivo de la Actividad Pesquera. Ministerio de la Producción. Oficina de Estudios Económicos, Enero 2017.

Roa R., B. Ernst \& F. Tapia. 1999. Estimation of size at sexual maturity: an evaluation of analytical and resampling procedures. Fishery Bulletin 97:570 - 580.

Rochet M.J. \& V.M. Trenkel. 2003. Which community indicators can measure the impact of fishing? A review and proposals. Canadian Journal of Fisheries and Aquatic Sciences 60(1), 86-99. https://doi.org/10.1139/f02-164

Simons R.A. 2011. ERDDAP - The Environmental Research Division's Data Access Program.'. Pacific Grove, CA, NOAA/ NMFS/SWFSC/ERD.

Torrejón-Magallanes J. 2017. sizeMat: Estimate Size at Sexual Maturity. R package version 0.3.0. [https://CRAN.R-project. org/package=sizeMat].

Trippel E.A \& H.H. Harvey. 1991. Comparison of methods used to estimate age and length of fishes at sexual maturity using populations of white sucker (Catostomus commersoni). Canadian Journal of Fisheries and Aquatic Sciences, 48(8), 1446 - 1459. https://doi.org/10.1139/f91-172

Trippel E.A. 1995. Age at maturity as a stress indicator in fisheries. Bioscience, 45(11), 759-771. https://doi.org/10.2307/1312628

Wootton R.J. 1984. Strategies and tactics in fish reproduction. In "Fish Reproduction: Strategies and Tactics". (Eds GW Potts and RJ Wootton.) pp. 1-12.

Yoneda M., M. Yamamoto, T. Yamada, M. Takahashi \& Y. Shima. 2015. Temperature-induced variation in sexual maturation of Japanese anchovy Engrauilis japonicus. Journal of the Marine Biological Association of the United Kingdom, 95(6), 1271 - 1276. https://doi.org/10.1017/ S0025315415000405 
